\title{
AN EXTENSION OF THE THEORY OF COMPLETELY DECOMPOSABLE TORSION-FREE ABELIAN GROUPS
}

BY

\author{
FRED RICHMAN $^{1}$
}

\begin{abstract}
We construct a class of strongly indecomposable finite rank torsion-free groups that includes the rank-one groups, and develop a complete set of invariants for them and their direct sums.
\end{abstract}

0. Introduction. Perhaps the only class of countable torsion-free abelian groups, for which a satisfactory complete set of invariants has been developed, is the class of countable completely decomposable torsion-free groups [FCHS, §85]. The theory of completely decomposable groups begins by classifying rank-one torsion-free groups up to isomorphism by their type, then shows that direct sums of rank-one groups are isomorphic only if they are composed of the same number of groups of each type. As an added bonus it turns out that summands of completely decomposable groups are completely decomposable.

We can attempt to generalize this elegant theory by starting with a larger class than the rank-one groups. A natural place to look is among the Butler groups: those groups that are isomorphic to pure subgroups of a finite direct sum of rank-one torsion-free groups [BTLR, ARNL]. In this paper we describe a class $\mathbf{C}$ of Bulter groups, that contains the rank-one groups and also contains strongly indecomposable groups of every finite rank, and exhibit a complete set of invariants for distinguishing among groups in C. Applying the global Azumaya theorems of [AHR] we also obtain a complete set of invariants for the class of direct sums of groups in C.

Let $n \geqslant 2$ be an integer. We shall be concerned with $n$-tuples $A=\left(A_{1}, \ldots, A_{n}\right)$ of subgroups $A_{i}$ of the rational numbers $Q$. For such an $A$ we define the rank- $(n-1)$ group $G(A)$ by

$$
G(A)=\left\{\left(a_{1}, \ldots, a_{n}\right) \in A_{1} \oplus \cdots \oplus A_{n}: \sum_{i=1}^{n} a_{i}=0\right\}
$$

This construction is slightly more general than it might appear at first glance. Any pure subgroup $K$ of $A_{1} \oplus \cdots \oplus A_{n}$ of corank one is the kernel of a nonzero

Received by the editors August 24, 1982.

1980 Mathematics Subject Classification. Primary 20K15, 20K20; Secondary 20K25, 20K40.

${ }^{1}$ Research supported by NSF grant MCS- 8003060 . 
homomorphism from $A_{1} \oplus \cdots \oplus A_{n}$ to $Q$ and so can be written as

$$
K=\left\{\left(a_{1}, \ldots, a_{n}\right) \in A_{1} \oplus \cdots \oplus A_{n}: \sum_{i=1}^{n} q_{i} a_{i}=0\right\}
$$

for suitable integers $q_{i}$. Replacing $A_{i}$ by $q_{i} A_{i}$ whenever $q_{i} \neq 0$ we see that $K$ can be written as a direct sum of a group of the form $G\left(A^{\prime}\right)$, where $A^{\prime}$ is an $m$-tuple of groups each equal to some $A_{i}$, and the direct sum of the remaining $n-m$ groups $A_{i}$.

If the types $\tau_{i j}^{A}$ of $A_{i} \cap A_{j}$ are incomparable for distinct pairs $\{i, j\}$, we call $A$ an $n$-tuple of doubly incomparable subgroups of $Q$. Let $\mathbf{C}$ denote the class of groups that are isomorphic to $G(A)$ for such an $A$. Taking $n=2$, and $A_{1}=A_{2}$ an arbitrary subgroup of $Q$, we see that $\mathbf{C}$ contains all rank-one torsion-free groups.

Given a doubly incomparable $n$-tuple $A$ of subgroups of $Q$ we construct two quantities. The first is a set of types $T_{A}$; the second is a matrix $E_{A}$ of integers and symbols $-\infty$, whose columns are indexed by $T_{A}$, and whose rows are indexed by the primes. The set $T_{A}$ is a complete set of invariants for $G(A)$ under quasi-isomorphism (Theorem 1.3). The matrix $E_{A}$ is determined by $T_{A}$ up to a finite number of entries, and together with $T_{A}$ forms a complete set of invariants for $G(A)$ under isomorphism.

We will have occasion to employ three operations on an $n$-tuple $A$ of subgroups of $Q$ that do not change the isomorphism class of $G(A)$. The first, permutation, replaces $A_{i}$ by $A_{\sigma(i)}$ for some permutation $\sigma$ of $\{1, \ldots, n\}$. The second, scalar multiplication, replaces each $A_{i}$ by $q A_{i}$ for a fixed nonzero rational number $q$. We denote the resulting $n$-tuple by $q A$. Clearly, multiplication by $q$ induces an isomorphism from $A_{1} \oplus \cdots \oplus A_{n}$ to $q A_{1} \oplus \cdots \oplus q A_{n}$ that takes $G(A)$ isomorphically onto $G(q A)$.

The third operation on $A$, called trimming, replaces each $A_{i}$ by $A_{i}^{\prime}=A_{i} \cap \sum_{j \neq i} A_{j}$. As $A_{i}^{\prime}$ is the image of the projection of $G(A)$ into $A_{i}$, it is clear that $G\left(A^{\prime}\right)=G(A)$. Moreover, $A_{i}^{\prime} \cap A_{j}^{\prime}=A_{i} \cap A_{j}$ so $\tau_{i j}^{A}=\tau_{i j}^{A^{\prime}}$ whence $A^{\prime}$ is doubly incomparable if $A$ is. We call $A^{\prime}$ the trimmed version of $A$ and say that $A$ is trimmed if $A=A^{\prime}$. It is easy to show that $A^{\prime}$ is trimmed, and is, in fact, the largest trimmed $n$-tuple contained in $A$.

Let $A$ and $B$ be $n$-tuples of subgroups of $Q$. Call $A$ and $B$ equivalent if they can be made equal via a succession of trimmings, permutations, and scalar multiplications. An equivalence class of $n$-tuples may be thought of as a sort of multiple type. We shall show that the following three conditions are equivalent if $A$ and $B$ are doubly incomparable.

(1) $A$ is equivalent to $B$,

(2) $G(A)$ is isomorphic to $G(B)$,

(3) $T_{A}=T_{B}$ and $E_{A}=E_{B}$.

We have already observed that (1) implies (2). Theorem 2.1 shows that (3) implies (1), and Theorems 1.3 and 2.3 show that (2) implies (3).

In $\$ 3$ we consider the case where each type in $T_{A}$ is the type of a subring of $Q$; in this case we can replace $E_{A}$ by an $n$-tuple of positive integers. In $\$ 4$ we consider groups that are quasi-isomorphic to groups in $\mathbf{C}$ and show that if, at each prime, $K$ is isomorphic to some group in $\mathbf{C}$, then $K$ is in $\mathbf{C}$ (Theorem 4.4). This result, in conjunction with Theorem 1.5 which states that the endomorphism rings of groups 
in $\mathbf{C}$ are subrings of $Q$, implies that the class of direct sums of groups in $\mathbf{C}$ is closed under the taking of summands. In $\S 5$ we apply the general theory of $[\mathbf{A H R}]$ to get a complete set of invariants for direct sums of groups in $\mathbf{C}$.

1. Types and quasi-isomorphism. Let $A$ be an $n$-tuple of subgroups of $Q$. Let $\tau_{i}^{A}=\sup \left\{\tau_{i j}^{A}: j \neq i\right\}=\operatorname{type}\left(A_{i} \cap \sum_{j \neq i} A_{j}\right)$, and let $T_{A}=\left\{\tau_{i}^{A}: i=1, \ldots, n\right\}$ where we think of $T_{A}$ as a multiset if some of the $\tau_{i}$ are equal. If $A$ is doubly incomparable we shall show how to compute $T_{A}$ from $G(A)$, and show that $T_{A}$ forms a complete set of invariants for $G(A)$ under quasi-isomorphism. For $I$ a nonempty subset of $\{1,2, \ldots, n\}$, let $\tau_{I}^{A}$ be the infimum of $\left\{\tau_{i}^{A}: i \in I\right\}$. When there is only one $n$-tuple $A$ under consideration, we will suppress the superscript $A$ on $\tau_{I}^{A}$. First we show how to retrieve the set of $\tau_{i j}$ from the type set of $G(A)$.

THEOREM 1.1. If $A$ is an $n$-tuple of subgroups of $Q$, then the type set of $G(A)$ consists of all $\tau_{l}$ with card $I \geqslant 2$. Thus, the maximal elements in the type set of $G(A)$ are precisely the $\tau_{i j}$.

Proof. The type of an element $x$ of $K$ is the infimum of the types of those $A_{i}$ in which $x$ has a nonzero coordinate. As the sum of the coordinates of $x$ is zero, $x$ cannot have precisely one nonzero coordinate. As $\bigcap_{i \in I} A_{i}$ is a nonzero subgroup, there is an element $x$ in $K$ such that $x$ has a nonzero coordinate in $A_{i}$ if and only if $i \in I$.

If $K$ is a group and $\tau$ is a type then $K(\tau)=\{x \in K: \operatorname{type}(x) \geqslant \tau\}$.

Lemma 1.2. Let $A$ be a doubly incomparable n-tuple of subgroups of $Q$, let $K=G(A)$ and let $M$ be the set of maximal types of elements of $K$. Let $\tau$ and $\tau^{\prime}$ be two distinct types in $M$. Then $K(\tau)+K\left(\tau^{\prime}\right)$ contains an element of type $\tau^{\prime \prime}$ in $M$ distinct from $\tau$ and $\tau^{\prime}$ if and only if $\tau=\tau_{i j}$ and $\tau^{\prime}=\tau_{j k}$ for some $i, j$ and $k$.

Proof. On the one hand, $K\left(\tau_{i j}\right)+K\left(\tau_{j k}\right)$ contains an element of type $\tau_{i k}$. On the other hand, if the pairs $\{i, j\}$ and $\{k, l\}$ are disjoint, then the nonzero elements of $K\left(\tau_{i j}\right)+K\left(\tau_{k l}\right)$ have type $\tau_{i j}, \tau_{k l}$ or $\inf \left(\tau_{i, j}, \tau_{k l}\right)$.

THEOREM 1.3. Let $A$ and $B$ be n-tuples of subgroups of $Q$. If $T_{A}=T_{B}$ then $G(A)$ and $G(B)$ are quasi-isomorphic. Conversely, if $A$ and $B$ are doubly incomparable, and $G(A)$ is quasi-isomorphic to $\left(i(B)\right.$, the'n $T_{A}=T_{B}$.

Proor. Supposc $T_{A} \quad T_{B}$. By trimming and permuting $A$ and $B$ we may assume that $B_{i}=q_{i} A_{i}$ for some rational numbers $q_{i}$. and we can then further assume that the $q_{i}$ are integers. Then $\left(i(B) \subset\left(i(A)\right.\right.$ and $G(A) / G(B) \subset\left(A_{1} / q_{1} A_{1}\right) \oplus \cdots \oplus$ $\left(A_{n} / q_{n} A_{n}\right)$ which is bounded by $q_{1} q_{2} \cdots q_{n}$.

Conversely, if $G(A)$ and $G(B)$ are quasi-isomorphic, then they have the same set $M$ of maximal types. Call two distinct elements of $M$ overlapping in $G(A)$ if they satisfy the condition of Lemma 1.2. As $G(A)$ and $G(B)$ are quasi-isomorphic, two elements of $M$ overlap in $G(A)$ if and only if they overlap in $G(B)$. The set $T_{A}=T_{B}$ consists of the suprema of maximal sets of pairwise overlapping elements of $M$.

The following example shows that the requirement that $A$ and $B$ be doubly incomparable in Theorem 1.3 cannot be removed, and suggests the problem of 
finding conditions on $T_{A}$ that allow $T_{A}$ to be read from (the quasi-isomorphism class of) $G(A)$.

EXAMPLE 1.4. Ler $p_{1}, p_{2}, p_{3}$ and $p_{4}$ be distinct primes, and let $R_{i}$ denote the ring generated over the integers by $1 / p_{i}$, and $R_{i j}$ denote $R_{i}+R_{j}$. Let

$$
A=\left(R_{12}, R_{34}, R_{14}, R_{23}\right) \text { and } B=\left(R_{12}, R_{34}, R_{13}, R_{24}\right) \text {. }
$$

Then $A$ and $B$ are trimmed 4-tuples of subgroups of $Q$ such that $T_{A} \neq T_{B}$, but we shall show that $G(A)$ and $G(B)$ are isomorphic. It suffices to show that $G(A)$ is isomorphic to $R_{1} \oplus \cdots \oplus R_{4}$ modulo the subgroup generated by $(1,1,1,1)$. Map $R_{1} \oplus \cdots \oplus R_{4}$ to $G(A)$ by taking $(w, x, y, z)$ to $(w-x, y-z,-w+z, x-y)$. The only difficulty is showing that this map is onto, and this can easily be done by localizing at each prime.

If $S$ is a set of types, we let $K(S)=\Sigma_{\tau \in S} K(\tau)$. If we let $\tau^{+}$denote the set of types greater than $\tau$, then $K\left(\tau^{+}\right)$is $K^{*}(\tau)$ in the usual notation. We denote the smallest type in a group $K$ by 0 . The following theorem shows that the groups in $\mathbf{C}$ are $B_{0}$-groups, that is, $K\left(\tau^{+}\right)$is pure for any type $\tau$ [ARNL, p. 14].

THEOREM 1.5. Let $A$ be a doubly incomparable $n$-tuple of subgroups of $Q$ and $K=G(A)$. If $\tau$ is a type, then

$$
\begin{aligned}
K\left(\tau^{+}\right) & =0 \quad \text { if } \tau=\tau_{i j} \text { for some } i \text { and } j, \\
& =K(\tau) \quad \text { otherwise. }
\end{aligned}
$$

Proof. As the $\tau_{i j}$ are the maximal types of elements in $K$, the first assertion is clear. If no element of $K$ has type precisely $\tau$ then the second assertion is clear. Otherwise $K(\tau)=G\left(A^{I}\right)$, where $A^{I}=\left\{A_{i}: i \in I\right\}$ for some subset $I$ of $\{1, \ldots, n\}$ of cardinality at least three, so it suffices to show that $K\left(0^{+}\right)=K$. We shall show that $K\left(0^{+}\right)_{p}=K_{p}$ for each prime $p$. Let $\left(a_{1}, \ldots, a_{n}\right)$ be an element of $K_{p}$ and choose $i \neq j$ such that $\left(A_{i}\right)_{p} \subset\left(A_{j}\right)_{p}$. For notational convenience we assume $i=1$ and $j=2$. Then

$$
\left(a_{1}, a_{2}, \ldots, a_{n}\right)=\left(a_{1},-a_{1}, 0, \ldots, 0\right)+\left(0, a_{1}+a_{2}, a_{3}, \ldots, a_{n}\right) .
$$

The first term is in $K\left(\tau_{12}\right)_{p} \subset K\left(0^{+}\right)_{p}$ and the second term is in $K\left(0^{+}\right)_{p}$ by induction $n$.

The requirement that $A$ be doubly incomparable in Theorem 1.5 cannot be dropped in light of the following example.

ExAmple 1.6. Define the 3-tuple $A$ of subgroups of $Q$ as follows. Let $A_{1}$ be the ring generated by $\frac{1}{2}$, let $A_{2}$ be the ring generated by $\frac{1}{3}$, and let $A_{3}$ be 5 times the ring generated by $\frac{1}{6}$. Then $K=G(A)$ is not a $B_{0}$-group because $K\left(0^{+}\right)_{5}$ is generated by $(5,0,-5)$ and $(0,5,-5)$ over the integers localized at 5 , but $K$ contains $(1,-1,0)$. Note that $A$ is trimmed and $K$ is quasi-decomposable.

The next theorem shows that the groups in $\mathbf{C}$ are candidates for the global Azumaya theory developed in [AHR].

THEOREM 1.7. If $A$ is a doubly incomparable $n$-tuple of subgroups of $Q$ and $K=G(A)$, then the endomorphism ring of $K$ is $\left\{x \in Q: x A_{i} \subset A_{i}\right.$ for $\left.i=1, \ldots, n\right\}$.

Hence, $K$ is strongly indecomposable. 
Proof. Let $\mathfrak{p}$ be an endomorphism of $K$. As $K\left(\tau_{i j}\right) \cong A_{i} \cap A_{j}$ is fully invariant, $\mathfrak{p}$ is multiplication by $x_{i j}$ on $K\left(\tau_{i j}\right)$ where $x_{i j}$ is a rational number such that $x_{i j} A_{i} \subset A_{i}$ and $x_{i j} A_{j} \subset A_{j}$. If $i, j$ and $k$ are distinct, then $K\left(\tau_{i j}\right)$ has nontrivial intersection with $K\left(\tau_{i j}\right) \oplus K\left(\tau_{j k}\right)$. Thus $x_{i j}=x_{j k}=x_{i k}$, so $\varphi$ is multiplication by a fixed element $x$ such that $x A_{i} \subset A_{i}$ for $i=1, \ldots, n$, on $K\left(0^{+}\right)=K$.

As $T_{A}$ determines the quasi-isomorphism class of $G(A)$, we should be able to find necessary and sufficient conditions on $T_{A}$ for $G(A)$ to be strongly indecomposable. More specifically, these conditions should be on the structure of the lattice generated by $T_{A}$.

2. The isomorphism invariants. For $x$ a nonzero rational number, and $p$ a prime, we let $v_{p} x$ denote the unique integer $m$ such that $x=p^{m} a / b$ where $a$ and $b$ are integers that are not divisible by $p$. If $A=\left(A_{1}, \ldots, A_{n}\right)$ is an $n$-tuple of subgroups of $Q$, and $p$ is a prime, we set

$$
e_{A}(i, p)=\inf \left\{v_{p} x: x \in A_{i}\right\} .
$$

Note that if $1 \in A_{i}$, then $-e_{A}(i, p)$ is the $p$-height of 1 in $A_{i}$. Also observe that $x$ is in $\left(A_{i}\right)_{p}$ if and only if $v_{p} x \geqslant e_{A}(i, p)$. Each $e_{A}(i, p)$ is either an integer or $-\infty$, and all but finitely many of them are nonpositive. The matrix $e_{A}$ completely determines $A$, and any such matrix is $e_{A}$ for some $A$.

We may think of the columns of the matrix $e_{A}$ as being indexed by $T_{A}$ rather than by $\{1, \ldots, n\}$ so that $e_{A}$ becomes independent of the particular ordering of the $n$-tuple $A$. If $T_{A}$ is a multiset, a case we are not particularly interested in, then there are minor technical problems in indexing $e_{A}$ by $T_{A}$ which we shall let the reader resolve. To construct the invariant $E_{A}$ we let $A^{\prime}$ be the trimmed version of $A$ and set

$$
E_{A}(\tau, p)=e_{A^{\prime}}(\tau, p)-\min \left\{e_{A^{\prime}}(\sigma, p): \sigma \in T_{A} \text { and } e_{A^{\prime}}(\sigma, p) \neq-\infty\right\} .
$$

Thus the $p$ th row of $E_{A}$ specifies the relative positions of the groups $\left(A_{i}\right)_{p}$ in $Q$.

Recall from the introduction that two $n$-tuples of subgroups of $Q$ are called equivalent if they can be made equal by a series of permutations, trimmings, and scalar multiplications.

Theorem 2.1. Let $A$ and $B$ be n-tuples of subgroups of $Q$. If $T_{A}=T_{B}$ and $E_{A}=E_{B}$, then $A$ is equivalent to $B$.

Proof. We may assume that $A$ and $B$ are trimmed. As $T_{A}=T_{B}$ we can, by permuting $B$, assume there are rational numbers $q_{i}$ so that $B_{i}=q_{i} A_{i}$. Multiplying $A$ by a suitable rational number we may assume that the $q_{i}$ are integers, that $v_{p} q_{i}=0$ if $E_{A}\left(\tau_{i}, p\right)=-\infty$, and that $v_{p} q_{i}=0$ for some $i$ such that $E_{A}\left(\tau_{i}, p\right)$ is finite, unless $E_{A}\left(\tau_{i}, p\right)=-\infty$ for all $i$. If after all that we have $v_{p} q_{i}>0$ for some $i$, then we can find $j$ such that $E_{A}\left(\tau_{j}, p\right)$ is finite and $v_{p} q_{j} \neq v_{p} q_{i}$. But then

$$
\begin{aligned}
E_{A}\left(\tau_{i}, p\right)-E_{A}\left(\tau_{j}, p\right) & =e_{A}(i, p)-e_{A}(j, p) \\
& \neq e_{B}(i, p)-e_{B}(j, p)=E_{B}\left(\tau_{i}, p\right)-E_{B}\left(\tau_{j}, p\right),
\end{aligned}
$$

contradicting $E_{A}=E_{B}$. Thus we may assume that $A=B$. 
LeMma 2.2. Let $A$ be a doubly incomparable $n$-tuple of subgroups of $Q$, let $p$ be $a$ prime, and let $K=G(A)$. If $e_{A}(2, p)$, and at least one of $e_{A}(1, p)$ and $e_{A}(3, p)$, are finite, then the cyclic group

$$
K\left(\tau_{12}, \tau_{23}, \tau_{13}\right)_{p} / K\left(\tau_{12}, \tau_{23}\right)_{p}
$$

has order $p^{m}$ where

$$
m=\sup \left\{e_{A}(1, p), e_{A}(2, p), e_{A}(3, p)\right\}-\sup \left\{e_{A}(1, p), e_{A}(3, p)\right\} .
$$

Proof. The group in question is isomorphic to $K\left(\tau_{13}\right)_{p} /\left(K\left(\tau_{13}\right)_{p} \cap K\left(\tau_{12}, \tau_{23}\right)_{p}\right)$. The $K\left(\tau_{i j}\right)_{p}$ are cyclic $Z_{p}$-submodules of $\left(A_{1} \oplus A_{2} \oplus A_{3}\right)_{p}$. If $v_{p} q_{i j}=$ $\sup \left(e_{A}(i, p), e_{A}(j, p)\right)$, then we have

$$
\begin{gathered}
K\left(\tau_{13}\right)_{p}=Z_{p}\left(q_{13}, 0,-q_{13}\right), \quad K\left(\tau_{12}\right)_{p}=Z_{p}\left(q_{12},-q_{11}, 0\right), \\
K\left(\tau_{23}\right)_{p}=Z_{p}\left(0, q_{23},-q_{23}\right) .
\end{gathered}
$$

Now $K\left(\tau_{13}\right)_{p} \cap K\left(\tau_{12}, \tau_{23}\right)_{p}=Z_{p}(r, 0,-r)$ where $v_{p} r=\sup \left\{e_{A}(i, p): i=1,2,3\right\}$ so $K\left(\tau_{13}\right)_{p} \cap K\left(\tau_{12}, \tau_{23}\right)_{p}=p^{m} K\left(\tau_{13}\right)_{p}$ where $m$ is as described.

We want to compute $E_{A}$ from $G(A)$. A prime $p$ is called relevant for $A$ if $\tau_{i}$ is finite at $p$ for at least two values of $i$. If $p$ is not relevant for $A$, then $E_{A}\left(\tau_{i}, p\right)=0$ if $\tau_{i}$ is finite at $p$. If $\tau_{i}$ is infinite at any prime $p$, then $E_{A}\left(\tau_{i}, p\right)=-\infty$. If $n=2$, then all finite entries of $E_{A}$ are 0 . Thus it suffices to compute $E_{A}\left(\tau_{i}, p\right)$ when $n \geqslant 3$ and $p$ is relevant and $\tau_{i}$ is finite at $p$.

THEOREM 2.3. Let $A$ be a doubly incomparable $n$-tuple of subgroups of $Q$ with $n \geqslant 3$, let $p$ be a relevant prime, and let $K=G(A)$. If $\tau_{i}$ is finite at $p$, then $E_{A}\left(\tau_{i}, p\right)$ is the supremum of the exponents of the cyclic groups

$$
K\left(\tau_{i j}, \tau_{i k}, \tau_{j k}\right)_{p} / K\left(\tau_{i j}, \tau_{i k}\right)_{p}
$$

where $j$ and $k$ range over elements of $\{1, \ldots, n\}$, different from $i$ and from each other, such that either $\tau_{j}$ or $\tau_{k}$ is finite at $p$.

Proof. Let $\mu_{i}$ denote the supremum in question. We may assume that $A$ is trimmed and, by multiplying $A$ by a suitable power of $p$, that $e_{A}(l, p)=E_{A}\left(\tau_{l}, p\right)$ for all $l$. If the pair $j, k$ is used in forming the supremum, then either $e_{A}(j, p)$ or $e_{A}(k, p)$ is nonnegative, so by Lemma 2.2 we see that $\mu_{i} \leqslant e_{A}(i, p)$. Moreover, if $e_{A}(i, p)>0$, then there exist $j$ and $k$ different from $i$ and from each other such that $e_{A}(j, p)$ and $e_{A}(k, p)$ are either 0 or $-\infty$, and at least one is 0 , so $\mu_{i}=e_{A}(i, p)$.

The following theorem characterizes those pairs $T$ and $E$ that are the invariants for some group in $\mathbf{C}$.

THEOREM 2.4. Let $n \geqslant 2$ be an integer, $T$ a set of $n$ types, and $E$ a matrix of integers and symbols $-\infty$ whose columns are indexed by $T$ and rows are indexed by the primes. Then there is a doubly incomparable n-tuple $A$ of subgroups of $Q$ such that $E=E_{A}$ and $T=T_{A}$ if and only if the following conditions hold.

(1) The pairwise infima of types in $T$ are incomparable.

(2) $\tau \leqslant \sup \left\{\tau^{\prime} \in T: \tau^{\prime} \neq \tau\right\}$ for each $\tau$ in $T$.

(3) $E(\tau, p)=-\infty$ if and only if $\tau$ is infinite at $p$. 
(4) Each row of E has at least two elements, and the least finite element, if any, is zero.

(5) If $B$ is an $n$-tuple of subgroups of $Q$ such that $T_{B}=T$, then for all but a finite number of primes $p$ there is an integer $k$ such that $E(\cdot, p)=e_{B}(\cdot, p)+k$.

Proof. Suppose $E=E_{A}$ and $T=T_{A}$. We may assume that $A$ is trimmed. The first condition holds because $A$ is doubly incomparable; the second follows from the definition of the $\tau_{i}$; the third, fourth and fifth from the definition of $E_{A}$. Conversely, suppose these five conditions hold. Because of condition (2) we can find $B$ satisfying the hypotheses of condition (5). We may assume that $B$ is trimmed, so every row of $e_{B}$ has at least two least elements. Let $S$ be the finite set of exceptional primes of condition (5). Replacing each $B_{i}$ by $q_{i} B_{i}$ where each $q_{i}$ is divisible only by those primes in $S$ we may assume that $e_{B}(\cdot, p)=E(\cdot, p)$ by condition (3). Set $A$ equal to the resulting $B$. Then condition (4) assures that $E=E_{A}$ and condition (1) makes $A$ doubly incomparable.

3. A special case. Let $A$ be a doubly incomparable $n$-tuple of subgroups of $Q$. If the types in $T_{A}$ are the types of subrings of $Q$, then these subrings are canonical representatives for their types and we can get a simpler looking set of invariants for $G(A)$.

THEOREM 3.1. Let $A$ and $B$ be trimmed doubly incomparable $n$-tuples of subgroups of $Q$ with $T_{A}=T_{B}$. If $A_{i}$ is a subring of $Q$ for $i=1, \ldots, n$, then there exist unique positive integers $q_{1}, \ldots, q_{n}$ such that:

(1) $G\left(q_{1} A_{1}, \ldots, q_{n} A_{n}\right) \cong G(B)$.

(2) For any prime $p$, at least two of the $q_{i}$ are not divisible by $p$.

(3) If $q_{i}$ is divisible by the prime $p$, then $p A_{i} \neq A_{i}$ and $p A_{j} \neq A_{j}$ for some $j \neq i$.

Proof. As $T_{A}=T_{B}$, and $A$ is an $n$-tuple of rings, $e_{B}$ has only finitely many entries different from 0 or $-\infty$. There exist unique positive integers $q_{1}, \ldots, q_{n}$ such that $q_{i}$ is not divisible by $p$ if $p A_{i}=A_{i}$ and setting $B^{\prime}=\left(q_{1} A_{1}, \ldots, q_{n} A_{n}\right)$ we have $e_{B^{\prime}}=E_{B}$. That the $q_{i}$ satisfy the required conditions follows from the properties of $E_{B}$; and since $E_{B}=E_{B^{\prime}}$ we have $G\left(B^{\prime}\right) \cong G(B)$.

If we further restrict $T_{A}$ to consist of finitely generated subrings of $Q$, then each $A_{i}$ is specified by the finite set $P_{i}$ of primes that are invertible in the ring $R_{i}$ of the same type, and an integer $q_{i}$ such that $A_{i}=q_{i} R_{i}$. Thus $G(A)$ is presented by specifying the finite sets $P_{1}, \ldots, P_{n}$ and the integers $q_{1}, \ldots, q_{n}$. It then makes sense to ask if there is an algorithm for deciding whether $G(A)$ is isomorphic to $G\left(A^{\prime}\right)$. In fact, Theorem 3.1 provides a simple algorithm for making that decision: we reduce the $n$-tuple $q_{1}, \ldots, q_{n}$ to the normal form defined in that theorem. Now an arbitrary pure subgroup $H$ of $R_{1} \oplus \cdots \oplus R_{n}$ may be specified by a matrix of integers $\left\{q_{i j}\right\}$ by setting $H=\left\{\left(a_{1}, \ldots, a_{n}\right): \sum q_{i j} a_{j}=0\right.$ for all $\left.i\right\}$. Does there exist a (good) algorithm for deciding when two such subgroups are isomorphic?

4. Local closure. Two torsion-free groups $G$ and $H$ are isomorphic at a prime $p$ if $G$ can be imbedded as a subgroup $G^{\prime}$ of $H$ such that $H / G^{\prime}$ is bounded by an integer not divisible by $p$. Let $C$ be a class of groups whose endomorphism rings are 
subrings of $Q$, and let $K$ be a group. Then the following are equivalent [AHR, Lemma 3.1].

(1) For each prime $p$ there is a group in $C$ that is isomorphic to $K$ at $p$.

(2) The endomorphism ring of $K$ is a subring of $Q$ and $K$ is isomorphic to a summand of a finite direct sum of groups in $C$.

If either of these two conditions holds we say that $K$ is locally in $C$. We say that $C$ is locally closed if, whenever $K$ is locally in $C$, then $K$ is in $C$. If $C$ is locally closed, then summands of direct sums of groups in $C$ are direct sums of groups in $C$ [AHR, Theorem B].

We shall show that the class $\mathbf{C}$ of groups isomorphic to $G(A)$ for some doubly incomparable $n$-tuple $A$ of subgroups of $Q$, is locally closed. Given $K$ locally in $\mathbf{C}$ we may restrict our attention to groups that are quasi-isomorphic to $K$. Every rank-one group is in $\mathbf{C}$, so we need only consider groups of rank two or more. As $K(\tau)$ is an additive functor of $K$, the local closure of the rank-two groups in $\mathbf{C}$ follows from the following theorem.

THEOREM 4.1. Let $A$ be a doubly incomparable triple of subgroups of $Q$ and let $K$ be a subgroup of finite index in $G(A)$. The $K=K\left(0^{+}\right)$if and only if $K=G\left(A^{\prime}\right)$ for some $A^{\prime}$.

Proof. Let $m$ be the index of $K$ in $G=G(A)$. We may assume that $A$ is trimmed, and that the $p$ th rows of $e_{A}$ and $E_{A}$ are the same for each prime $p$ dividing $m$. If $p$ is a prime dividing $m$ then, by replacing $A_{i}$ by $p^{-t} A_{i}$ if necessary, thereby possibly changing $m$ but not the set of primes dividing $m$, we can arrange for $e_{A}(i, p)$ to be 0 or $-\infty$ for each prime $p$ dividing $m$ and all $i$.

If $H$ is a subgroup of $G$ such that $G / H \cong Z_{p} \oplus Z_{p}$ then $H=p G$. Thus there is an integer $n$ so that $K \subset n G$ and $n G / K$ is cyclic. Hence we may assume $G / K$ is cyclic. If some irrelevant prime $p$ divides $m$, then the order of $G / p G$ does not exceed $p$, so $K \subset p G$. Thus we may assume that no irrelevant prime divides $m$. So we can write $K$ as

$$
K=\left\{\left(a_{1}, a_{2}, a_{3}\right) \in G: m \mid s_{1} a_{1}+s_{2} a_{2}+s_{3} a_{3}\right\}
$$

where $0 \leqslant s_{i}<m$. For $i, j, k$, a permutation of $1,2,3$, let $q_{k}$ be the GCD of $s_{i}-s_{j}$ and $m$. Then $K\left(\tau_{i j}\right)=\left(m / q_{k}\right) G\left(\tau_{i j}\right)$. Suppose the $s_{i}$ are all congruent modulo some prime $p$ dividing $m$. As $a_{1}+a_{2}+a_{3}=0$ we may assume that the $s_{i}$ are all congruent to 0 modulo $p$. But then $K$ would contain $(\mathrm{m} / \mathrm{p}) G$ and so $G / K$ would not be cyclic of order $m$. Thus the $q_{k}$ are pairwise relatively prime and $K\left(0^{+}\right) \subset$ $\left(m / q_{1} q_{2} q_{3}\right) G$.

We may assume that $s_{k}=0$. If $\left(a_{1}, a_{2}, a_{3}\right) \in K$, then $s_{i} a_{i}+s_{j} a_{j}$ is divisible by $m$. As $q_{i}$ divides $s_{j}$ and $m$, and $q_{i}$ and $s_{i}$ are relatively prime, we have $q_{i}$ divides $a_{i}$. Therefore,

$$
K \subset K^{\prime}=\left\{\left(a_{1}, a_{2}, a_{3}\right) \in G: a_{i} \in q_{i} A_{i} \text { for } i=1,2,3\right\} .
$$

As $q_{1}$ and $q_{2}$ are relatively prime we can find integers $x$ and $y$ such that $x q_{1}+y q_{2}=1$. Hence $\left(x q_{1}, y q_{2},-1\right)$ is in $G$ at all primes dividing $m$, and has order $q_{3}$ modulo $K^{\prime}$. Repeating this argument we see that $G / K^{\prime}$ has order at least $q_{1} q_{2} q_{3}$. In particular, if 
$K\left(0^{+}\right)=K$, then $q_{1} q_{2} q_{3}=m$, so $K=K^{\prime}$. The "only if" follows from Theorem 1.5.

Theorem 4.1 does not extend to the class $n=4$ as the following example shows.

EXAMPLE 4.2. Let $A$ be a doubly incomparable 4-tuple of subgroups of $Q$ and let $p$ be a prime such that $e_{A}(i, p)=0$ for $i=1, \ldots, 4$. Then there is a subgroup $K \subset G(A)$ of index $p$ such that $K=K\left(0^{+}\right)$but $K$ is not isomorphic to $G\left(A^{\prime}\right)$ for any $A^{\prime}$.

Proof. Define $K$ to be

$$
K=\left\{\left(a_{1}, a_{2}, a_{3}, a_{4}\right) \in G(A): p \mid a_{1}+a_{2}\right\} .
$$

The $p$ th row of $E_{A}$, computed from $K$ by Theorem 2.3 , is $(1,1,1,1)$ which is not possible, so $K$ cannot be isomorphic to $G\left(A^{\prime}\right)$ for any $A^{\prime}$. To see that $K\left(0^{+}\right)=K$, it suffices to show that $K\left(0^{+}\right)_{p}=K_{p}$ as $K_{q}=G$ for $q \neq p$. But $(1,-1,0,0),(0,0,1,-1)$ and $(0, p,-p, 0)$ are in $K\left(0^{+}\right)$and generate $K_{p}$ over $Z_{p}$.

Despite the fact that there are groups $K$ with $K=K\left(0^{+}\right)$that are quasi-isomorphic to a group in $\mathbf{C}$ but not isomorphic to such a group, it is still true that $\mathbf{C}$ is locally closed. To see this we first establish the following

Lemma 4.3. Let $A$ and $B$ be doubly incomparable n-tuples of subgroups of $Q$. Then $G(A)$ is isomorphic to $G(B)$ at the prime $p$ if and only if $T_{A}=T_{B}$ and the pth rows of $E_{A}$ and $E_{B}$ are the same.

Proof. If $K=G(A)$ and $L=G(B)$ are isomorphic at the prime $p$, then $T_{A}=T_{B}$ by Theorem 1.3. Moreover, $K(S)_{p} \cong L(S)_{p}$ for any set $S$ of types, so the $p$ th rows of $E_{A}$ and $E_{B}$ are the same by Theorem 2.3. Conversely, suppose that the $p$ th rows of $E_{A}$ and $E_{B}$ are the same. Then, upon trimming $A$ and $B$ and multiplying $A$ by an appropriate rational number, we may assume that the $p$ th rows of $e_{A}$ and $e_{B}$ are the same, and that $A \subset B$. Then $G(A) \subset G(B)$ and $G(B) / G(A)$ has order not divisible by $p$.

THEOREM 4.4. Let $\mathbf{C}$ be the class of groups $G(A)$ where $A$ is a doubly incomparable $n$-tuple of subgroups of $Q$. Then $\mathbf{C}$ is locally closed.

Proof. Suppose that, for each prime $p$, there is a group in $\mathbf{C}$ that is isomorphic to $K$ at $p$. By Lemma 4.3, there is a fixed group $G$ in $\mathbf{C}$ that is isomorphic to $K$ at each p. By [AHR, Lemma 3.5] $K$ is isomorphic to $G$.

COROllary 4.5. Let $\mathbf{C}$ be the class of groups $G(A)$ where $A$ is a doubly incomparable $n$-tuple of subgroups of $Q$. Then summands of direct sums of groups in $\mathbf{C}$ are isomorphic to direct sums of groups in $\mathbf{C}$.

Proof. By Theorem 4.4 the class $\mathrm{C}$ is locally closed. The result then follows from [AHR, Theorem B].

5. The class of direct sums. As we have a complete set of invariants for groups in $\mathrm{C}$, and the endomorphism rings of these groups are principal ideal domains, we can construct a complete set of invariants for direct sums of groups in $\mathbf{C}$ by utilizing [AHR, Theorem D]. A direct sum of groups in $\mathbf{C}$ may be specified by a family $\mathbb{Q}$ of 
doubly incomparable tuples of subgroups of $Q$; we denote the direct sum of the groups $G(A)$ for $A$ in $\mathscr{Q}$ by $G(\mathscr{Q})$. We associate with $\mathscr{Q}$ the multiset $\sigma_{\mathscr{Q}}=\left\{T_{A}\right.$ : $A \in \mathbb{Q}\}$ and for each $T$ in $\mathcal{T}_{\mathbb{Q}}$ and prime $p$ the multiset $\mathcal{E}_{\mathbb{Q}}(T, p)=\left\{E_{A}(\cdot, p): A \in \mathbb{Q}\right.$ and $\left.T_{A}=T\right\}$ of functions from $T$ to $Z \cup\{-\infty\}$. Notice that the multiset $\varepsilon_{Q}$ does not record the fact that the functions $E_{A}(\cdot, p)$ and $E_{A}\left(\cdot, p^{\prime}\right)$ come from the same $A$ in $Q$. Hence, we cannot recover the family $\{G(A): A \in \mathbb{Q}\}$, up to isomorphism, from $\mathcal{T}_{\mathscr{Q}}$ and $\mathcal{E}_{\mathscr{Q}}$. This reflects the fact that nonisomorphic multisets of groups in $\mathbf{C}$ can give rise to isomorphic direct sums.

THEOREM 5.1. Let $Q$ and $\mathbb{Q}^{\prime}$ be families of doubly incomparable tuples of subgroups of $Q$. Then $G(\mathbb{Q})$ is isomorphic to $G\left(\mathbb{Q}^{\prime}\right)$ if and only if $\mathcal{J}_{\mathscr{Q}}=\mathcal{J}_{\mathbb{Q}^{\prime}}$ and $\mathcal{E}_{\mathbb{Q}}=\mathcal{E}_{\mathbb{Q}^{\prime}}$.

Proof. By [AHR, Theorem D] the groups $G(\mathscr{Q})$ and $G\left(\mathbb{Q}^{\prime}\right)$ are isomorphic if and only if, for each $C$ in $\mathbf{C}$ and prime $p$, there is a bijection between $\{A \in \mathbb{Q}: C$ is isomorphic to $G(A)$ at $p\}$ and $\left\{A^{\prime} \in Q^{\prime}: C\right.$ is isomorphic to $G\left(A^{\prime}\right)$ at $\left.p\right\}$. If $C$ is isomorphic to $G\left(A^{\prime \prime}\right)$, then, by Lemma $4.3, C$ is isomorphic to $G(A)$ at $p$ if and only if $T_{A}=T_{A^{\prime \prime}}$ and $E_{A}(\cdot, p)=E_{A^{\prime \prime}}(\cdot, p)$.

The next theorem characterizes those pairs $\mathcal{T}$ and $\mathcal{E}$ that are the invariants of a direct sum of groups in $\mathbf{C}$.

THEOREM 5.2. Let $\mathcal{T}$ be a multiset of finite sets of types, and for each $T$ in $\mathcal{T}$ and prime $p$ let $\mathcal{E}(T, p)$ be a multiset of functions from $T$ to $Z \cup\{-\infty\}$. Then there is $a$ multiset $Q$ of doubly incomparable tuples of subgroups of $Q$ such that $\sigma=\sigma_{Q}$ and $\mathcal{E}=\mathcal{E}_{A}$ if and only if the following conditions hold.

(1) Each $T$ in $T$ has cardinality at least 2 and consists of types whose pairwise infima are incomparable.

(2) If $T \in \mathcal{T}$ and $\tau \in T$, then $\tau \leqslant \sup \{\sigma: \tau \neq \sigma \in T\}$.

(3) If $T \in \mathcal{T}$ with multiplicity $m \neq 0$, then $\mathcal{E}(T, p)$ has cardinality $m$, and if $E(\cdot, p)$ is in $\mathcal{E}(T, p)$, then

(i) $E(\tau, p)=-\infty$ if and only if $\tau$ is infinite at $p$, and

(ii) $E(\cdot, p)$ takes on its least value at least twice, and its least finite value is 0 .

(4) If $B$ is an n-tuple of subgroups of $Q$ such that $T_{B}=T$, and $k$ is a cardinal less than the multiplicity $m$ of $T$ in $\mathcal{T}$, then for all but a finite number of primes $p$ the function $E_{B}(\cdot, p)$ appears more than $k$ times in $\mathcal{E}(T, p)$.

Proof. The necessity of the first three conditions follows immediately from Theorem 2.4. To see that condition (4) is necessary, suppose there are infinitely many primes such that $E_{B}(\cdot, p)$ appears no more than $k$ times in $\mathcal{E}(T, p)$. Then there are $m$ elements $A$ of $\mathscr{Q}$ such that $T_{A}=T$ and $E_{B}(\cdot, p) \neq E_{A}(\cdot, p)$, contradicting Theorem 2.4, condition (5).

To show sufficiency we may assume that $\mathcal{T}$ consists of a single type $T$ with multiplicity $m$. Because of conditions (1) and (2) we can choose a doubly incomparable $n$-tuple $A$ of subgroups of $Q$ such that $T_{A}=T$, and by Theorem 2.4 we can arrange for $e_{A}(\cdot, p)$ to be a specified element of $\mathcal{E}(T, p)$ for a finite number of primes $p$. Using condition (4) we can collect such $A$ into the required family $\mathscr{Q}$. 


\section{REFERENCES}

[ARNL] D. Arnold, Pure subgroups of completely decomposable groups, Abelian Group Theory, Lecture Notes in Math., vol. 874, Springer-Verlag, Berlin and New York, 1981, pp. 1-31.

[AHR] D. Arnold, R. Hunter and F. Richman, Global Azumaya theorems in additive categories, J. Pure Appl. Algebra 16 (1980), 223-242.

[BTLR] M. C. R. Butler, A class of torsion-free abelian groups of finite rank, Proc. London Math. Soc. 15 (1965), 680-698.

[FCHS] L. Fuchs, Infinite abelian groups, Vol. II, Academic Press, New York, 1973.

Department of Mathematics, New Mexico State University, Las Cruces, New Mexico 88003 